\title{
Dissecção coronária espontânea e Infarto Agudo do Miocárdio no puerpério: um verdadeiro desafio para o cardiologista de intervenção
}

Miguel Bernardino Antunes Vicente 1*, Luis Mariano de Lima Domingos 1, Roger Ravelo Dopico 1, Tomás Carlos Mendés Peralta 1, Gelson Rodrigues Pereira 1, José Ribeiro Bunda Ricardo 1.

${ }^{1}$ Unidade de Diagnóstico e Intervenção Cardiovascular, Clínica Girassol, Luanda, Lda, Angola.

*Corresponding author: Miguel Bernardino Antunes Vicente. Street: Comandante Gika, no225 - Maianga. Zip Code: 12345-123 - Luanda, Lda, Angola. Phone: +244 940980920. E-mail: miguelantunesv@gmail.com.

Research Ethics Committee Approval (if necessary): Declaramos que a paciente aprovou o estudo assinando um termo de consentimento informado e que o estudo seguiu as diretrizes éticas estabelecidas pela Declaração de Helsinque.

Received on: Jul 26, 2021. Accepted on: Aug 02, 2021. Available online: Aug 6, 2021.

\section{Resumo}

A Dissecção coronária espontânea é uma rara causa de síndrome coronária aguda não relacionada com a aterosclerose, diagnosticada em $20-30 \%$ das mulheres em idade reprodutiva antes dos 50 anos. Desde sua primeira descrição há mais de meia década por Harold Pretty, vem sendo palco de inúmeros desafios quanto ao diagnóstico precoce e a melhor abordagem terapêutica. A coronariografia constitui o padrão-ouro no diagnóstico dessa entidade e o tratamento conservador mostra-se seguro, e a intervenção coronária percutânea está associada a altas taxas de insucesso. Apresentamos o caso de uma puérpera angolana de 41 anos diagnosticada com dissecção coronária espontânea tratada de forma conservadora.

Palavras-chave: Puérpera; Dissecção coronária; Síndrome coronária aguda, Infarto Agudo do Miocárdio.

\section{Introdução}

A dissecção coronária espontânea (DCE) é uma rara condição clínica associada a síndrome coronária aguda (SCA) frequentemente diagnosticada em mulheres saudáveis em idade reprodutiva [1]. É definida como uma separação espontânea, não traumática ou iatrogênica, das paredes arteriais coronárias com ou sem ruptura [2]. Foi descrita pela primeira vez em 1930 por Harold Pretty [3]. 
A sua prevalência tem sido descrita como elevada, entre 20-30\% especialmente no periodo peri e pósparto, porém, diagnosticados geralmente na autópsia, pois em sua maioria falecem sem diagnóstico antemmortem [1,4-5].

Constitui a principal causa de Infarto Agudo do Miocárdio (IAM) em 1 de 5 mulheres menores de 50 anos no periodo gestacional, e apresenta altas taxas de morbi-mortalidade [6].

Vários autores relatam a influência multifactorial (físicos e emocionais) para DCE como causa predisponente das arteriopatias que podem ser $o$ substrato de enfraquecimento da parede arterial, aumentando a vulnerabilidade para dissecção, facto que representa um dilema potencialmente fatal entre as mulheres em idade fértil $[1,4,7]$.

Vale ressaltar a associação entre DCE e as anormalidades arteriais não coronárias, onde a displasia fibromuscular é a mais frequentemente documentada dentre elas, e está associada a piores prognósticos [8].

Esta condição foi por muitos anos subdiagnosticada, e com a melhoria do conhecimento do perfil angiográfico e o advento de novas modalidades de imagem intracoronária, o diagnóstico melhorou substancialmente impactando significativamente no aumento de reportes de várias séries nos últimos anos [2,9].

A coronariografia constitui o padrão-ouro no diagnóstico dessa entidade, por meio deste, é evidenciado um falso lúmen formado pelas camadas da parede arterial coronária gerando compressão externa do lúmen coronário verdadeiro, restringindo o fluxo coronário, justificando o quadro clínico típico de SCA nesses pacientes [10].

O tratamento conservador mostra-se seguro e com excelentes resultados evolutivos, exceto para pacientes instáveis de alto risco, uma vez que a intervenção coronária percutânea (ICP) no momento da DCE está associada a altas taxas de insucesso, e a maioria das dissecções regridem espontaneamente [2,9]. Este relato tem o objectivo de descrever o caso de uma puérpera angolana de 41 anos diagnosticada com dissecção coronária espontânea tratada de forma conservadora.

\section{Relato de Caso}

Trata-se de uma paciente angolana de 41 anos de idade, com história obstétrica de G3P3A0C0, actualmente no puerpério de parto eutócico de recém-nascido do sexo masculino de 3.810 gramas sem complicações no pós-parto durante 3 semanas pós alta, altura que deu início da sintomatologia caracterizada por precordialgia súbita, opressiva e intensa de \pm 12 horas de evolução.

Com esse quadro, a paciente foi assistida pelo serviço de urgência em 
uma clínica particular com estabilidade hemodinâmica (pressão arterial: 110/70mmHg; frequência cardíaca: 87bpm; frequência respiratória de 16irpm; temperatura: $36,5^{\circ} \mathrm{C}$ e saturação de O2). Realizou-se o eletrocardiograma que evidenciou sinais sugestivos de IAM de parede anterior (Figura 1).

Posteriormente, a paciente foi submetida a coronariografia que evidenciou a dissecção coronária da artéria descendente anterior (Figura 2). Decidiu-se tratamento conservador (dupla antiagregação plaquetária e betabloqueador) pelo contexto hemodinâmico estável da puérpera e pelo alto risco de complicações relacionado a ICP nesse grupo de pacientes.

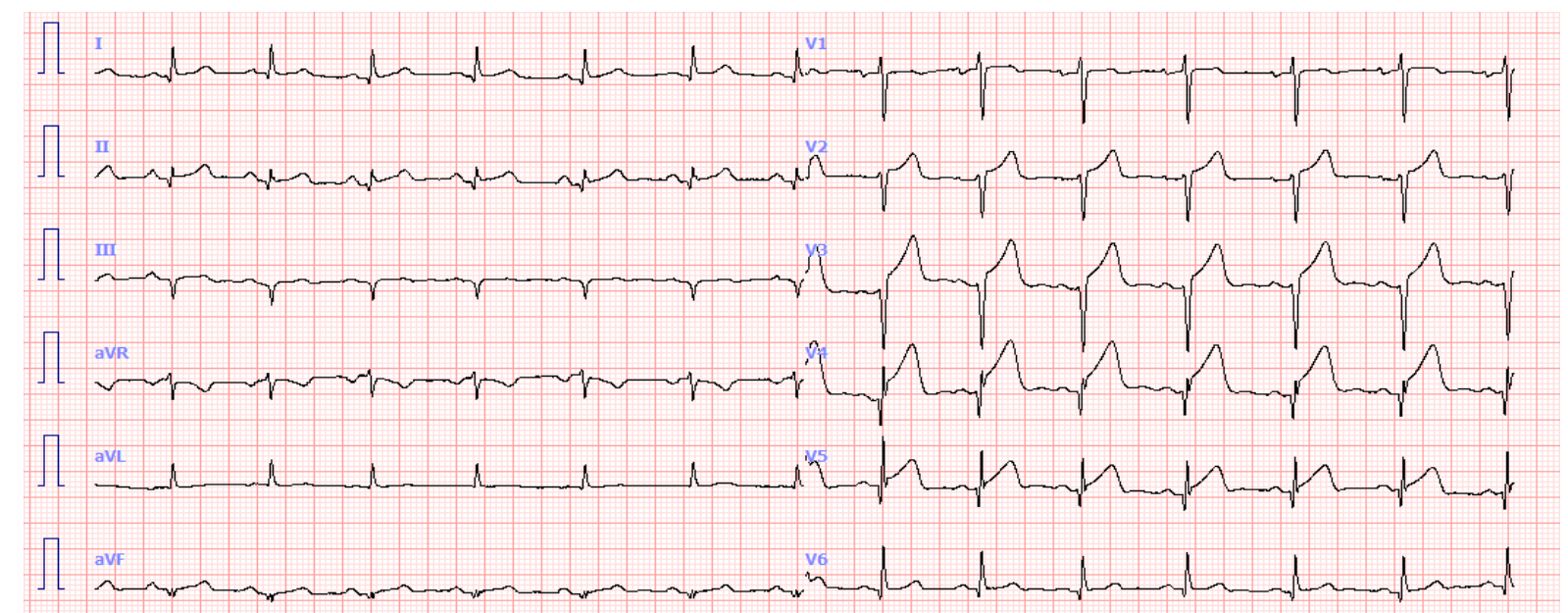

Figure 1. Eletrocardiograma de 12 derivações. Ritmo sinusal, Infarto de parede anterior (V1-V6).
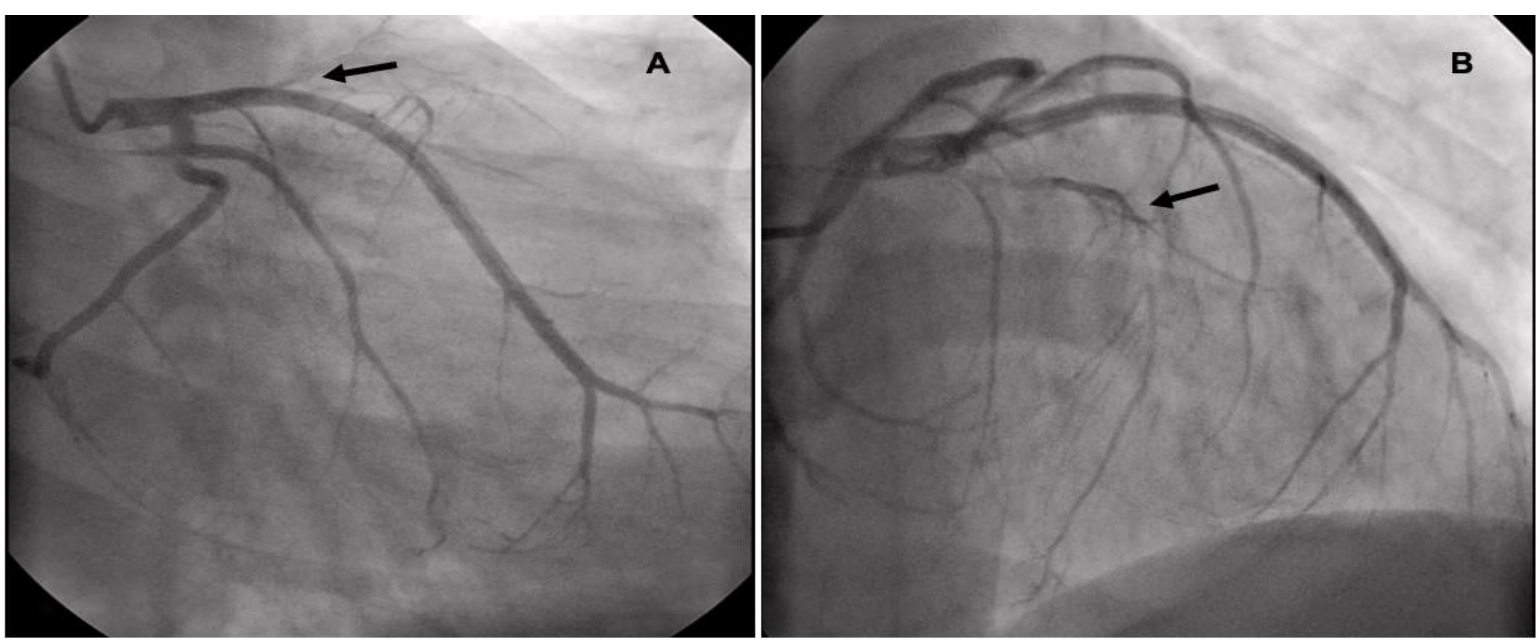

Figura 2. Coronariografia realizada pela via da artéria radial direita. A: Vista octogonal oblíqua anterior direita caudal $25 \mathrm{com} 30^{\circ}$ e B: Vista octogonal oblíqua anterior direita com cranial $15 \mathrm{com} 35^{\circ}$, onde se observa ousão no segmento proximal da ADA (setas). 


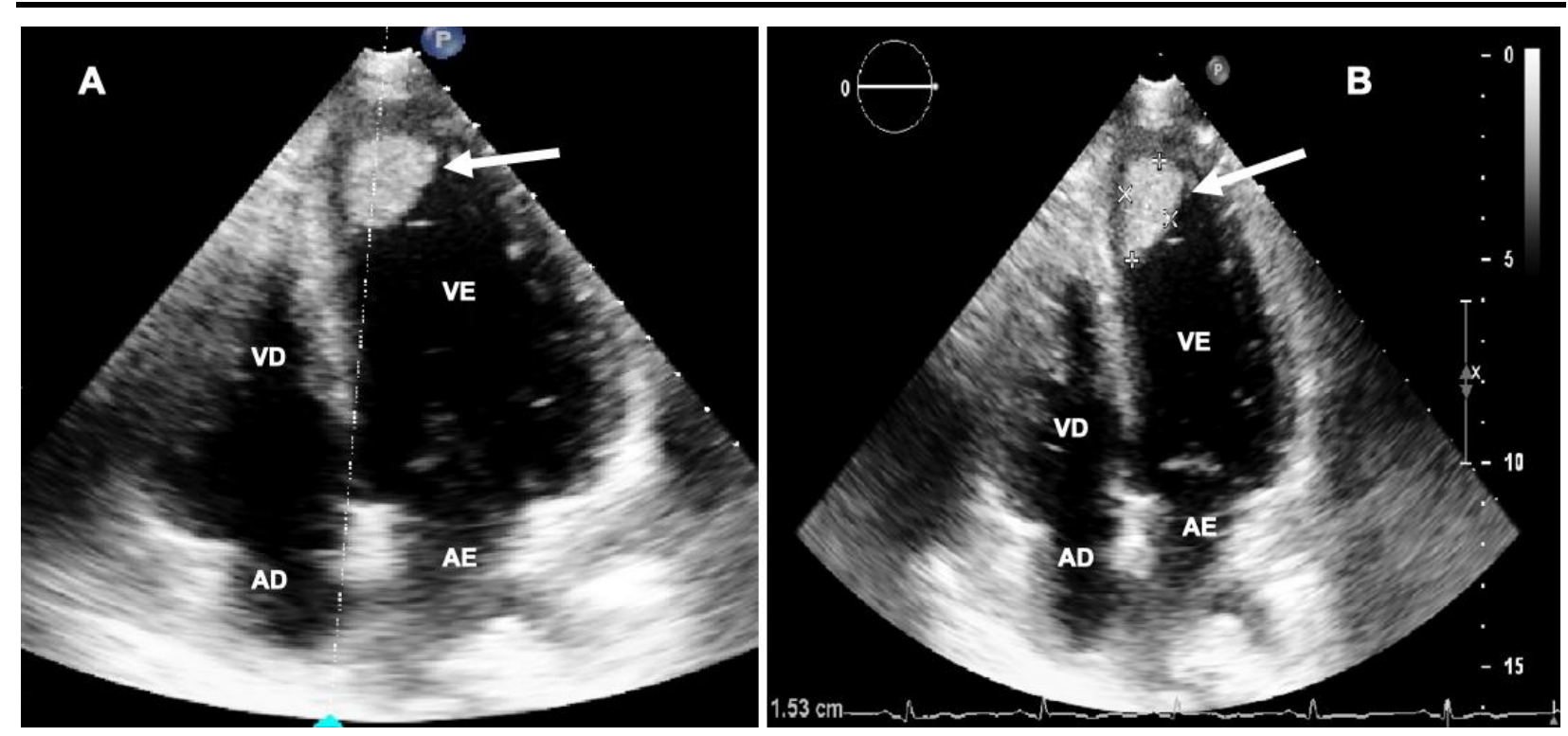

Figura 3. Ecocardiograma transtorácico. A e B - observa-se trombo apical (seta).

No angioTac das artérias aterosclerótica nem imagem inequívoca coronárias na descendente anterior não endoluminal suspeita da presença de foi obervada nenhuma lesão dissecção vide (figura 4).

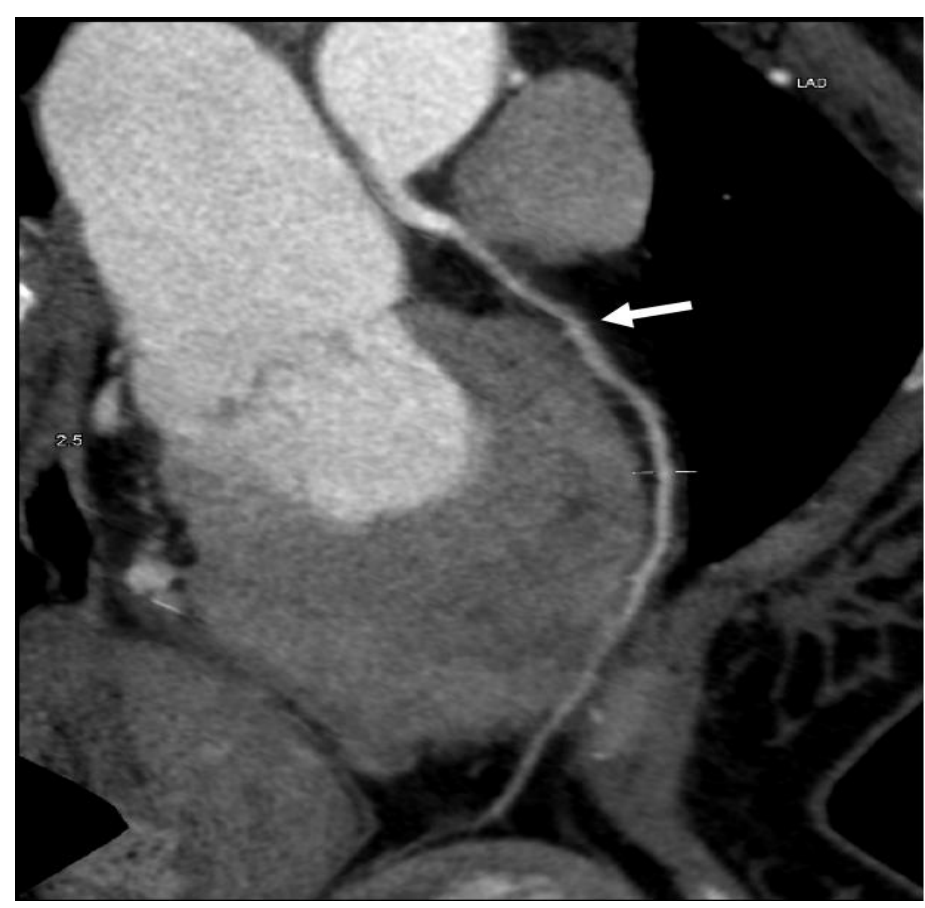

Figura 4. AngioTac das coronárias, sem evidência de lesão endoluminal sugestiva de dissecção (seta). 


\section{Discussão e Conclusão}

A DCE é uma causa rara de SCA não relacionada a aterosclerose diagnosticada geralmente em mulheres em idade reprodutiva antes dos 50 anos $[6,8]$. Esta condição está frequentemente associada as anormalidades arteriais onde a mais comum é a displasia fibromuscular [8,11].

A real prevalência dessa entidade se mantém um desafio uma vez que maioritariamente o seu diagnóstico é feito post-morten. De qualquer modo, estima-se que ela se aproxime aos $4 \%$ de todos os SCA e aos $20-30 \%$ nas mulheres em idade fértil [5,9].

Mecanismos como provável ruptura de vasa vasorum e/ou dissecção da camada íntima favorecem a criação de um espaço intramural e consequentemente o acúmulo de sangue proporcionando um falso lúmen que é responsável por diminuir o calibre arterial coronário, reduzindo o fluxo sanguíneo, desiquilibrando a demanda e oferta de nutrientes aos cardiomiócitos [5].

$\mathrm{O}$ advento de tecnologias inovadoras como a tomográfica de coerência óptica (OCT), ultrassom intravascular (IVUS) e a angiotomografia têm permitido o aprimoramento substancial do diagnóstico uma vez que fornece detalhes intraluminal da parede coronária, mesmo assim, a coronariografia se mantém como Gold standard apesar da sua limitação (lumenográfica bidimensional) principalmente no contexto de urgência por ser o métodos mais disponível recomendado para abordagem inicial de DCE $[9,10]$.

Além do mais, permite classificala em três tipos: Tipo 1 refere-se à aparência clássica de múltiplos lúmens radiolúcidos ou coloração de contraste da parede arterial; tipo 2 refere-se à presença de estenose difusa que pode ser de gravidade e comprimento variáveis, com subvariante A: quando a estenose está delimitada por segmentos proximais e distais ao hematoma intramural e a subvariante B: o estreitamento difuso que se estende até a ponta distal da artéria, e o tipo 3: é a estenose focal ou tubular que mimetiza a aterosclerose, geralmente usamos a IVUS ou a OCT para confirmar seu diagnóstico $[9,12]$.

A paciente em questão apresentou uma dissecção tipo 2, confirmada por coronariografia de urgência, e decidiu-se como estratégia inicial de tratamento, o conservador, pela estabilidade hemodinâmica da puérpera. Embora seja ainda palco de inúmeras incertezas e receios, a estratégia inicial de tratamento da DCE diferente do SCA aterosclerótico tem seu maior risco de complicação relacionado com a ICP (que apresenta 
taxas altas de dissecção coronária iatrogênica, dificuldade em orientar a guia no lúmen verdadeiro, colocação de stent no falso lúmen), por isso, é quase consensual, no momento decidir pela abordagem conservadora, desde que em pacientes elétrica e hemodinamicamente estáveis, sem lesão do tronco da artéria coronária esquerda e sem isquemia ativa [11,12].

Quando há instabilidade hemodinâmica e clínica (choque, isquemia contínua e recorrente), instabilidade elétrica (alterações evolutivas e dinâmicas do eletrocardiograma, arritmias ventriculares graves) e dissecção de tronco da coronária esquerda deve-se urgentemente considerar a ICP ou cirurgia de revascularização do miocárdio $[9,12]$.

Em um estudo recentemente publicado que fez uma revisão sobre a DEC mostrou que o tratamento conservador tem benefícios incertos principalmente naqueles não tratados com stents [5], por outro lado, numa série de casos envolvendo 33 pacientes revelou que $82 \%$ destes receberam o tratamento conservador com dupla antiagregação por um periodo de 17 meses e que foi um preditor de bom prognóstico para os pacientes com DCE [7].

No caso em questão, apesar da estabilidade clínica evidenciada, o ecocardiograma evolutivo mostrou trombo apical organizado, o que motivou o início da hipocoagulação, apesar dos riscos subjacentes. Embora seja controverso o uso da anticoagulação em pacientes com DCE pelo risco de extensão da dissecção, vale ressaltar seus benefícios acrescidos na resolução do trombo em VE e melhoria na permeabilidade luminal verdadeira [13]. No cenário clínico em causa, a presença de trombo em VE aumentaria o risco de embolização sistémica com eventuais complicações devastadoras para a paciente, justificando a adição do anticoagulação oral com resultado satisfatório.

A DCE é uma causa rara e subdiagnosticada de Síndrome coronária aguda associada ao periodo reprodutivo, com repercussões letais quando não identificada precocemente. Apesar das inovações nos métodos de imagens para auxiliar e precisão do diagnóstico, a coronariografia se mantém como Gold standard. O tratamento conservador é recomendado quase consensualmente em relação a ICP pelas altas taxas de insucesso desta, porém deve ser individualizado em determinadas circunstâncias.

\section{Referências}

[1] Tweet MS, Hayes SN, Codsi E, Gulati R, Rose CH, Best PJ. Dissecção espontânea de artéria coronária associada à gravidez. Journal of the 
American College of Cardiology. 2017; 70 (4):426-435.

[2] Saw J, Starovoytov A, Humphries K, Sheth T, So D, Minhas K, Brass N, Lavoie A, Bishop H, Lavi S, Pearce C, Renner S, Madan M, Welsh RC, Lutchmedial S, Vijayaraghavan R, Aymong E, Har B, Ibrahim R, Gornik HL, Ganesh S, Buller C, Matteau A, Martucci G, Ko D, Mancini GBJ. Canadian spontaneous coronary artery dissection cohort study: in-hospital and 30-day outcomes. Eur Heart J. 2019 Apr 14;40(15):1188-1197. doi: 10.1093/eurheartj/ehz007.

[3] Pretty, HC. Dissecting aneurysm of coronary artery in a woman aged 42: rupture. BMJ. 1931;1: 667.

[4] Kavalar R, Krajnc I. Spontaneous dissecting aneurysm of the coronary artery. A rare cause of myocardial infarction and sudden death. Wien Klin Wochenschr. 2003 May 30;115(10):34750. doi: 10.1007/BF03041487.

[5] Yip A, Saw J. Spontaneous coronary artery dissection-A review. Cardiovasc Diagn Ther.2015; 5 (1): 37-48. doi: 10.3978 / j.issn.2223-3652.2015.01.08

[6] Lobo AS, Cantu SM, Sharkey SW, Grey EZ, Storey K, Witt D, Benson G, Garberich RF, Kubota Y, Bairey Merz $\mathrm{CN}$, Henry TD. Revascularization in Patients With Spontaneous Coronary Artery Dissection and ST-Segment Elevation Myocardial Infarction. J Am
Coll Cardiol. 2019 Sep 10;74(10):12901300. doi: 10.1016/j.jacc.2019.06.065.

[7] Bastante T, García-Guimaraes $\mathrm{M}$, Muñiz M, Cuesta J, Rivero F, Antuña P, De Rueda C, Hernández-Muñiz $S$, Aguilar R, Salamanca J, Pozo-Osinalde $\mathrm{E}$, Jiménez-Borreguero J, Batlle $\mathrm{M}$, Friera A, Alfonso F. Manejo contemporáneo de la disección coronaria espontánea. REC Interv Cardiol. 2020;2:247-255. doi: 10.24875/RECIC.M20000095.

[8] Agrawal V, Kim ESH. Spontaneous Coronary Artery Dissection: Cardiac Manifestations of Vascular Disease. Prog Cardiovasc Dis. 2018 MarApr;60(6):629-634. doi: 10.1016/j.pcad.2018.04.001.

[9] Hayes SN, Kim ESH, Saw J, Adlam $\mathrm{D}$, Arslanian-Engoren C, Economy KE, Ganesh SK, Gulati R, Lindsay ME, Mieres JH, Naderi S, Shah S, Thaler DE, Tweet MS, Wood MJ; American Heart Association Council on Peripheral Vascular Disease; Council on Clinical Cardiology; Council on Cardiovascular and Stroke Nursing; Council on Genomic and Precision Medicine; and Stroke Council. Spontaneous Coronary Artery Dissection: Current State of the Science: A Scientific Statement From the American Heart Association. Circulation. 2018 May 8;137(19):e523e557.

doi:

$10.1161 /$

CIR.0000000000000564. 
[10] Al-Hussaini, A, Adlam D. Spontaneous coronary artery dissection, Heart, (2017). 103(13), 1043-1051.

[11] Kotecha D, David A. Spontaneous coronary artery dissection: new insights on diagnosis and management.

[12] Yogeswaran V, Ramakrishna S, MacGregor JS, Zier L, Goldschlager N. Dor torácica associada à gravidez: um caso de dissecção espontânea da artéria coronária. Case Rep Cardiol. 202115 de janeiro; 2021: 4057182. doi: 10.1155 / 2021/4057182.

[13] Santana M, Guragai N, Patel B, Vasudev R, Randhawa P, Joshi M. et al. Dissecção espontânea da artéria coronária associada ao achado incidental de trombo ventricular esquerdo. Journal of Community Hospital Internal Medicine Perspectives. 2021;11(2):249-252. doi: 10. 1080 / 20009666. 2021. 1891689.

Conflict of interest: The author declares no conflicts of interest.

Agradecimentos: Dr ${ }^{a}$. Elsa Fernandes e Dro Telmo Martins (Ecocardiografistas) e Droํ․ Jorge Conde Marquez (Ginecoobstetra).

Financiamento: Não se aplica.

Como citar este artigo: Vicente MBA, Domingos LML, Dopico RR, Peralta TCM, Pereira GR, Ricardo JRB. Dissecção coronária espontânea e Infarto Agudo do Miocárdio no puerpério: um verdadeiro desafio para o cardiologista de intervenção. Brazilian Journal of Case Reports. 2021 Jul-Sep; 01(3):95-102. 